\title{
Exemplar-Based Image Completion Model Employing PDE Corrections
}

\author{
Jiying WU, Qiuqi RUAN, Gaoyun AN \\ Institute of Information Science, Beijing Jiaotong University \\ Room 621, Institute of Information Science, Beijing Jiaotong University \\ e-mail:happywjy82@gmail.com,qqruan@center.njtu.edu.cn,gyan@bjtu.edu.cn
}

Received: July 2008; accepted: April 2009

\begin{abstract}
To suitably complete an image without seams, block effects, and artifacts, a novel exemplar-based image completion model employing partial differential equation (PDE) is proposed. Firstly, the novel model determines the processing order of exemplar according to a composite function, which is the product of the colour property and structure property in exemplar. Then the exemplar along a geometric structure is processed prior to other parts of the image. Secondly, the most similar exemplar is found in the CIELAB colour space, and the size of it is adaptively determined by the local textured information. Thirdly, a Poisson equation is adopted to remove the seams, block effects, and artifacts in the image generated by the exemplar-based model. Finally, a bi-directional diffusion PDE is used to assist the completion of lathy linear structure. Experimental results demonstrate that the novel model can properly reconstruct the target region while preserving the geometric structure without inducing block effects, which leads to its better performance than the conventional exemplar-based image completion models.
\end{abstract}

Keywords: exemplar-based image completion, adaptive size of exemplar, partial differential equation, correcting artifacts.

\section{Introduction}

Removing objects and restoring damaged image are important image processing tasks. Given an input image with selected target region, both the geometric property and color information should be propagated from the known parts of image into the target region. However, the removed target region is selected by user, and then the completion procedure is executed automatically and seamless.

There are three main approaches related to image completion. The first one is image inpainting. The concept of image inpainting is firstly proposed by Bertalmio et al. (2000). It uses an along isophote diffused partial differential equation (PDE) to propagate the known image information into the target region. PDE updates a pixel's gray value based on the geometric information in the adjacent domain (Wu et al., 2009). It preserves the linear structure and smoothes the region contour. When the target region is small, the visual perception of the reconstructed image is good. PDE processes image only according to the local information, so when the target region is large or textured, the processed result is bad. PDE cannot reconnect the linear structure in a large region and cannot restore 
texture patterns. There are many PDE-based inpainting models (Chan and Shen, 2001; Bertalmio et al., 2001; Bertalmio, 2006). All of these models are suitable for completing the small and non-textured target region. They would over-smooth the image with a large target region.

Texture synthesis is another kind of image completion approach. It regenerates texture patterns in the target region. It aims at reconstructing the texture patterns properly and seamlessly (Tran and Datta, 2003; Ng et al., 2005; Efros and Leung, 1999; Efros and Freeman, 2001). The texture synthesis approach uses variable neighborhood searching to preserve the entire texture pattern (Tran and Datta, 2003). Graphcut method is used to reduce seams between texture patches. It generates $\omega$-tile set to avoid highly repetitive patterns (Ng et al., 2005). A non-parametric method based on Markov random field theory is introduced to preserve the local structure and produce a wide variety of textures (Efros and Leung, 1999). It is extended to the exemplar-based non-parametric model to accelerate the computing speed (Efros and Freeman, 2001). The texture synthesis approach processes image according to the global information, so the entire texture pattern in image is resorted. The texture synthesis approach is completely unrelated to the local geometric property; therefore, it cannot preserve the geometric structure in image. The texture synthesis approach is not suitable for the geometric natural image completion.

Efros et al. (2001) proposed the exemplar-based texture synthesis model. Then Bornard et al. (2002) introduced this model into the natural image completion. Perez et al. (2004) further proved that used in geometric image completion this approach could generate good result. In exemplar-based image completion model, the basic unit of synthesis is a filling patch (exemplar) more than a single pixel. An exemplar is compared and copied during the completion procedure. Harrison determines the filling order by "textureness" of pixel (Harrison, 2001): the pixels which are highly constrained by neighborhood pixels have higher filling priority. Although this intent is good, strong linear structures cannot always be properly preserved. The method proposed by Drori and Yeshurun (2003) "iteratively approximates the unknown region and fills in the image by adaptive fragments". It uses an inverse alpha matte as the confidence map to determine the traversal order. This method is time-consuming and could not often generate good result. Sun et al. (2005) introduced an image completion model with structure propagation. User should mark the linear structure in the target region firstly and then the image patch is copied along the prescribed direction. This model generates a good completed image but it needs user's interaction. Criminisi et al. (2004) proposed an exemplar-based image completion model which determines the filling order under the constraint of PDE-based data item. The exemplar which is along geometric linear structure has a higher filling priority, and the geometric property in the image is preserved. In Komodakis and Tziritas (2006), a completion model which restores the target region by minimizing a global objective function is proposed. The completed image generated by this model has no artifact. The exemplarbased approach achieves good performances used in natural image completion with large target regions. But this approach does not consider the local geometric property; therefore, it could not preserve the linear structures in image as well. Also, it cannot restore the complex geometric structures in natural image completion. 
In this paper a novel exemplar-based image completion model is proposed. The whole image domain is $D$, the target region domain to be removed or restored is $\Omega$, and image information in $D-\Omega$ is known. Firstly, the processing order of exemplar is determined by its color property and structure property simultaneously. The color property is the known information in the exemplar with an adaptively determined size, and the structure property is the result of a cross-isophote diffused PDE which diffuses considering the extent of edge. The exemplar along a geometric structure has a high processing priority, and it is restored prior to other parts of the image. Secondly, the most similar exemplar is found in the CIELAB color space which is used to describe the colors visible to human eye. The size of the exemplar is adaptively determined by the textured information to preserve more texture patterns. Thirdly, a Poisson equation is adopted to correct the completed image. It reduces seams and block effects in the completed image. It also removes the artifacts caused by the greedy exemplar-based model. Finally, a bi-directional diffused PDE is used to assist the completion procedure of the image which has some lathy linear structures to be completed.

The novel exemplar-based model proposed in this paper is novel because it uses a cross-isophote diffused PDE to constrain the processing order; therefore, it has a good linear structure preserving property. The size of exemplar is dynamically determined by the local textured information; the seams and block effects are removed by the PDE. Because the exemplar-based model could not be used for complex geometric structures completion, the novel model adopts a bi-directional diffused PDE to assist the completion procedure. Then the novel model could be used to restore the natural image with both large target regions and complex geometric structures.

The remainder of this paper is organized as follows: Section 2 will give deep analysis about the novel exemplar-based image completion model; in Section 3 the validity of the novel model is confirmed by experimental results on different testing images; and Section 4 will give a conclusion for our work.

\section{Exemplar-Based Completion Model}

\subsection{Determining the Processing Order of Exemplar}

The processing order of exemplar affects the completion result (Drori and Yeshurun, 2003; Sun et al., 2005; Criminisi et al., 2004). In this paper, the processing order is determined by both the colour and structure property of the exemplar.

The colour property is computed according to the known colour information in the exemplar with a proper size. The ordinary values of the sizes are $3 \times 3,4 \times 4$ and $5 \times 5$. While in this paper, the right size of the exemplar is adaptively determined by the textured information of image. An initial size is appointed firstly, and the size is $4 \times 4$. Then the sizes of all exemplars are computed according to the textured information. The statistical property of the textured information is

$$
T\left(\Psi_{p}\right)=\frac{\sum_{\xi \in \Psi_{p}} f(I(\xi)-\mu)}{N\left(\Psi_{p}\right)},
$$


where $\Psi_{p}$ is the exemplar, the size of which is computed based on pixel $p, T\left(\Psi_{p}\right)$ is the statistical property of textured information in exemplar $\Psi_{p}, \xi$ denotes the pixel in $\Psi_{p}$, $I(\xi)$ is the color value of $\xi, \mu$ is the average color value of all pixels in $\Psi_{p}, f$ computes the color difference between isolated pixel and the average result of pixels in $\Psi_{p}, N\left(\Psi_{p}\right)$ is the number of pixels in $\Psi_{p}$. Initially, $N\left(\Psi_{p}\right)$ is 16 .

The computed $T\left(\Psi_{p}\right)$ is then compared with textured thresholds $\lambda_{1}, \lambda_{2}\left(\lambda_{1}<\lambda_{2}\right)$. The thresholds are determined experimentally and they are the variables which $30 \%$ and $60 \%$ computed $T\left(\Psi_{p}\right)$ are smaller than, respectively. If $\Psi_{p}$ is textured, $T\left(\Psi_{p}\right)$ is larger than $\lambda_{2}$. To preserve the entire textured information, the size of $\Psi_{p}$ is $5 \times 5$. If $\Psi_{p}$ is nontextured, $T\left(\Psi_{p}\right)$ is small and the size is small enough to not copy error textured patterns. The size is $3 \times 3$. Detailed description is shown in Algorithm 1:

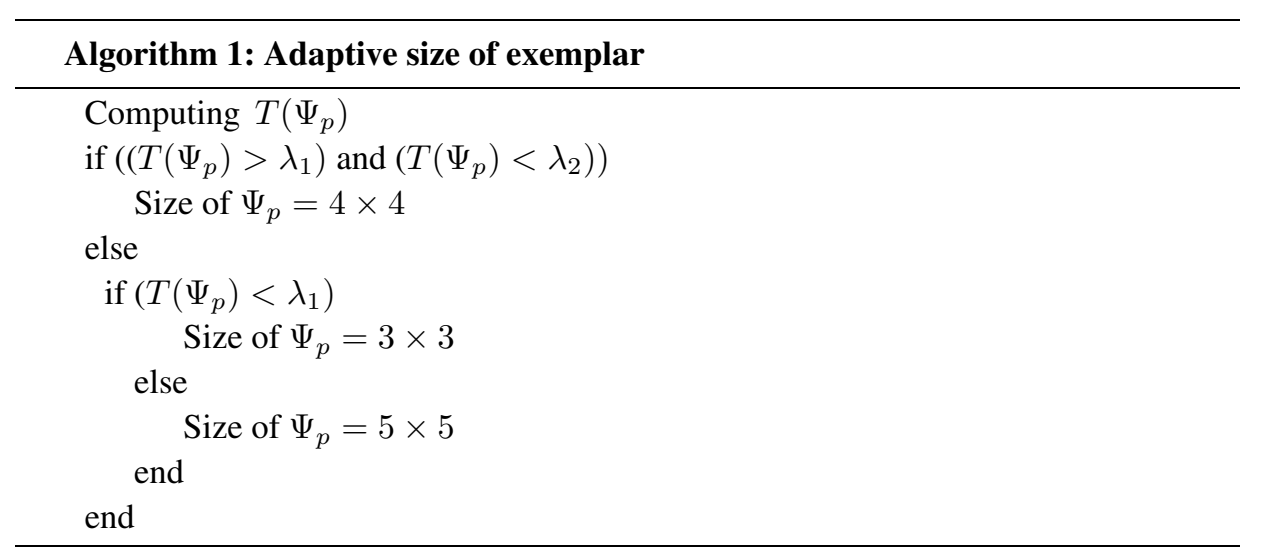

Then the colour property of exemplar $\Psi_{p}$ is computed by the known colour information in it:

$$
C P\left(\Psi_{p}\right)=\frac{\sum_{q \in \Psi_{p}} C(q)}{\left|\Psi_{p}\right|},
$$

where $C(\cdot)$ denotes the confidence value in image. Initially, the values of $C(\cdot)$ for the known pixels in $D-\Omega$ are $1 \mathrm{~s}$, and for the unknown pixels in $\Omega$ are 0 s. The size of exemplar $\left(\left|\Psi_{p}\right|\right)$ is determined according to the above analysis. The confidence values of all pixels in $\Psi_{p}$ are added and then the colour property $C P\left(\Psi_{p}\right)$ of exemplar $\Psi_{p}$ is generated.

The structure property of an exemplar is determined by the geometric information. So it is computed by using a directional diffused PDE in this paper. The PDE is

$$
\begin{aligned}
& I(\xi)_{t}=\frac{1}{|\nabla I(\xi)|} I(\xi)_{\vec{t}} \vec{t} \\
& I(\xi)_{\vec{t}} \vec{t}=\frac{I(\xi)_{x}^{2} I(\xi)_{y y}+I(\xi)_{y}^{2} I(\xi)_{x x}-2 I(\xi)_{x} I(\xi)_{y} I(\xi)_{x y}}{|\nabla I(\xi)|^{2}} \\
& \vec{t}=\nabla^{\perp} I(\xi)
\end{aligned}
$$


where $I(\xi)$ denotes the color value of pixel $\xi$ in exemplar, $x, y$ are space reference frame, $I(\xi) \vec{t} \vec{t}$ is the second order directional derivative along isophote $\vec{t}$. In an image, the color values of pixels along an isophote are constant. The color value along $\vec{t}$ is denoted as $I(\xi) \equiv \lambda, \mathrm{d} s$ is the length element along an isophote

$$
\mathrm{d} \lambda / \mathrm{d} s=|\nabla I(\xi)| .
$$

That is, $|\nabla I(\xi)|$ denotes the change rate of geometric structure cross isophotes. So the diffusion of Eq. (3) depends on the contrast between isophotes, and it considers the extent of edge. It computes the color values along isophotes, so the exemplar along a geometric structure has a large value of structure property. To determine the processing order, the value of structure property should be positive, so it is computed as

$$
S P\left(\Psi_{p}\right)=\sum_{\xi \in \Psi_{p}} \frac{1}{|\nabla I(\xi)|}|I(\xi) \vec{t} \vec{t}| .
$$

The composite function which is used to determine the processing order of exemplar is

$$
F\left(\Psi_{p}\right)=C P\left(\Psi_{p}\right) S P\left(\Psi_{p}\right) .
$$

So the exemplar which has the largest $F()$ will be processed and restored firstly.

Here we give some experimental results to demonstrate the importance of the processing order. There is a geometric linear structure in Fig. 1(a), and it is partially occluded by a randomly selected region in Fig. 1(b). Three completion methods are used to restore the image, respectively. Fig. 1(c) shows the completion procedure of the model which has no special function determine the processing order. This model completes the target region from outside to inside. It completes the outmost layer firstly, so it could not preserve the geometric structure and there are artifacts in the completed image. Fig. 1(d) shows the completed images which are processed by the model proposed in Criminisi $e t$ al. (2004). This model uses a confidence item and a data item to constrain the processing order. The sizes of exemplars which determine the confidence items are constant. The data item is determined by an along-isophote diffused PDE. This PDE diffuses without considering the extent of edge, and it often causes error diffusions. So in Fig. 1(d), the model fills some isotropic regions prior to parts of the geometric line, and the geometric structure is not thoroughly preserved. Fig. 1(e) is the completion procedure of the model which determines the processing order by the composite function. The processing order is adaptively determined by the color property of exemplar. The composite function uses a cross-isophote diffused PDE to determine the structure property. There is no error diffusion in this PDE and it diffuses considering the extent of edge. The geometric line is well preserved in Fig. 1(e) and there is no artifact.

There is a similar experiment in Criminisi et al. (2004). In that experiment, the model could preserve geometric structure well when the line in image is occluded by a regular region. We redo this experiment and the results are shown in Fig. 2. The geometric 


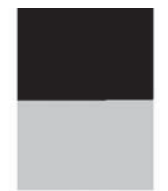

(a) original image

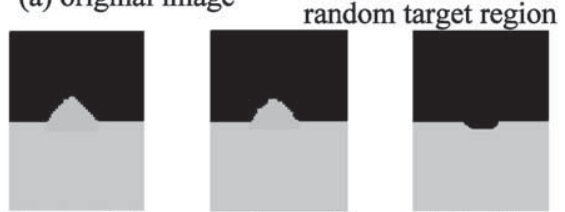

(c) completion procedure of the model with no

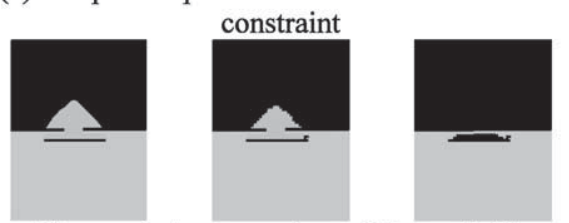

(d) completion procedure of the model in

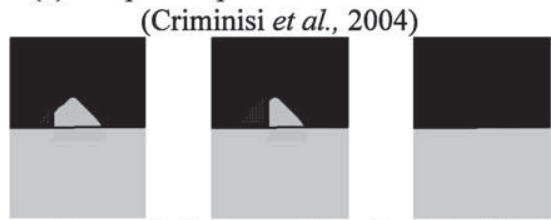

(e) completion procedure of our model

Fig. 1. Images with random target region processed by exemplar-based models.

structure in Fig. 2(c) is still not preserved. Fig. 2(d) is the result of the model in Criminisi et al. (2004). It preserves the line. Fig. 2(e) is the result of our model which preserves the linear structure too. The results in Fig. 1(d) and Fig. 2(d) are different, because the occluded region in Fig. 2(b) is a regular semi-ellipse, while that in Fig. 1(b) is only a randomly selected region. The model which uses the composite function to determine the processing order has a better geometric structure preserving property.

Experimental results in Fig. 1 and Fig. 2 demonstrate that our model preserves geometric structure well even when the target region is selected randomly. Also it is shown the completed result greatly relies on the selected target region.

\subsection{Finding the Most Similar Exemplar}

When the processing orders of all exemplars are determined, the exemplar $\Psi_{H}$ which has the highest processing priority is found. The size of $\Psi_{H}$ is adaptively determined by local textured information analyzed in Section 2.1. An exemplar in the known parts of image with the same size as $\Psi_{H}$ is then found to fill in the target region.

$$
\Psi_{\bar{q}}=\arg \min _{\Psi_{q} \in D-\Omega} d\left(\Psi_{H}, \Psi_{q}\right)
$$




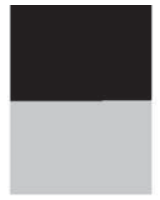

(a) original image

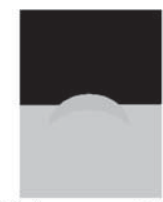

(b) image with a regular target region

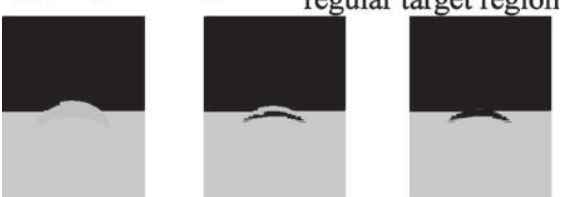

(c) completion procedure of the model with

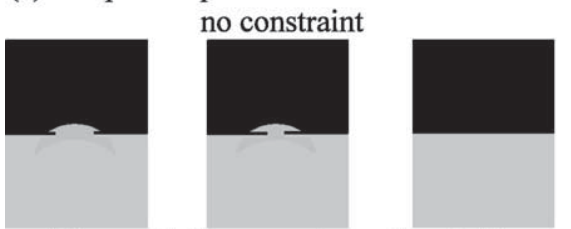

(d) completion procedure of model in

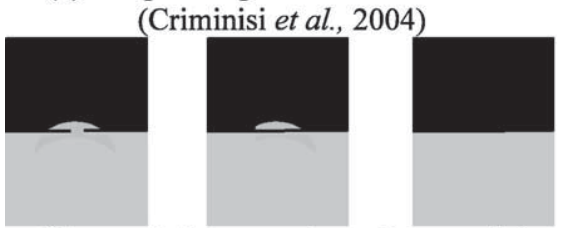

(e) completion procedure of our model

Fig. 2. Images with regular target region processed by exemplar-based models.

where $\Psi_{\bar{q}}$ is the exemplar in the known parts of image and it is most similar to $\Psi_{H}$ based on the color similarity. $d\left(\Psi_{H}, \Psi_{q}\right)$ is the color distance between the known information in two exemplars. In this paper, the exemplar distance $d(\cdot)$ is computed in the CIELAB color space.

The CIELAB is used to describe the color visible to human eyes. It has the perceptual uniformity, and its L component matches human perception of lightness. The color difference between two pixels in the CIELAB is computed by treating each pixel as a point in the 3-D space:

$$
D\left(p_{1}, p_{2}\right)=D\left(L_{1}, L_{2}\right) D\left(a_{1}, a_{2}\right) D\left(b_{1}, b_{2}\right), \quad p_{1} \in \Psi_{H}, p_{2} \in \Psi_{q}
$$

where $\left(L_{1}, a_{1}, b_{1}\right)$ and $\left(L_{2}, a_{2}, b_{2}\right)$ represents the colors of two pixels $p_{1}, p_{2}$, respectively. The color distances of all the pixels in two exemplars are summed up to generate $d\left(\Psi_{H}, \Psi_{q}\right)$ :

$$
d\left(\Psi_{H}, \Psi_{q}\right)=\sum_{p_{1} \in \Psi_{H}, p_{2} \in \Psi_{q}} D\left(p_{1}, p_{2}\right)
$$


Euclidean Distance is used to weigh the similarity between images:

$$
D\left(p_{1}, p_{2}\right)=\left(L_{2}-L_{1}\right)^{2}+\left(a_{2}-a_{1}\right)^{2}+\left(b_{2}-b_{1}\right)^{2}, \quad p_{1} \in \Psi_{H}, p_{2} \in \Psi_{q} .
$$

When all the color distances between exemplars are computed, the exemplar which has the smallest distance is the most similar one for the exemplar $\Psi_{H}$. It is filled into the target region, and the confidence value $C(\cdot)$ of all pixels in exemplar $\Psi_{H}$ should be updated with

$$
C(p)=C(\bar{p}), \quad \forall p \in \Psi_{H} \cap \Omega .
$$

\subsection{A Poisson Equation Correction}

In this paper, a Poisson equation is used to correct the completion procedure to reduce the seams, block effects and artifacts in the result of the exemplar-based model. In mathematics, Poisson equation is a PDE, it is

$$
\Delta \varphi=f
$$

where $\Delta$ is the Laplace operator, and $f$ and $\varphi$ are real or complex-valued functions on a manifold. When the manifold is a 2-D image domain, the Laplace operator $\Delta$ is denoted as $\nabla^{2}$ and then the Poisson equation is written as

$$
\nabla^{2} \varphi=f
$$

The most similar exemplar $\Psi_{\bar{q}}$ should be filled into the target region, and both the color information and textured information are filled. While, some pixels in $\Psi_{H}$ are already known, that is, the color values of these pixels affect the filled values. When $\Psi_{\bar{q}}$ is filled to overlap $\Psi_{H}$, this operation should be implemented under the constraint of a Poisson equation:

$$
\min _{I(\xi)} \iint_{\Psi_{\bar{q}}}|\nabla I(\xi)|^{2} \quad \text { s.t. }\left.\quad J(\xi)\right|_{\Psi_{H}}=\left.I(\xi)\right|_{\Psi_{\bar{q}}}
$$

where $I(\xi)$ denotes the color value of pixel in $\Psi_{\bar{q}}$. These values are different from the original values in $\Psi_{\bar{q}}$ and they are the constrained results of the Poisson equation. $J(\xi)$ represents the existing values of the known pixels in $\Psi_{H}$. The boundary condition of the Poisson equation is that the filled values in $\Psi_{\bar{q}}$ should be consistent with the existing values in $\Psi_{H}$. It is proved image processing in the Total Variation (TV) domain could preserve geometric structure (Chan and Shen, 2001), so in this paper, Eq. (14) is implemented in TV domain as

$$
\min _{I(\xi)} \iint_{\Psi_{\bar{q}}}|\nabla I(\xi)| \quad \text { s.t. }\left.\quad J(\xi)\right|_{\Psi_{H}}=\left.I(\xi)\right|_{\Psi_{\bar{q}}} .
$$


For the simplicity of implementation, Poisson Eq. (14) is written in a guidance field form:

$$
\min _{\hat{I}(\xi)} \iint_{\Psi_{\bar{q}}}|\nabla \hat{I}(\xi)| \quad \text { s.t. } \quad I(\xi)-\left.J(\xi)\right|_{\Psi_{H}}=\left.\hat{I}(\xi)\right|_{\Psi_{\bar{q}}} .
$$

In Eq. (16), the divergence value between two exemplars is minimized, and this value is computed under the constraint of the original difference value in $\Psi_{H}$.

When an exemplar is filled into the target region, a Poisson equation is executed. There are two reasons for adopting a Poisson equation to correct the completion result.

- The completion method proposed in this paper is implemented based on the exemplar, that is, the basic unit of synthesis is an exemplar more than a single pixel, and the processing speed is fast. But this operation induces severe seams between exemplars. There are block effects in the completed image. Poisson equation is used to minimize the divergence between exemplars, and it is a good image fusion method. So with the Poisson equation, seams between exemplars are removed. The seamless image has a better visual perception.

- The exemplar-based model is greedy: once a source exemplar is copied into the target region, it will not change. If the filled exemplar is not consistent with the known information in adjacent exemplars, there are artifacts in the completed image. For the greedy method, the artifacts will not be removed, and the visual perception of the completed image is bad. While if the Poisson equation is used, every exemplar is filled under the constraint of the known information, and the divergence value between them is minimized. So the difference between them is small, and there is no artifact in the image. The experimental results in the next section will demonstrate that the completed results of the model which adopts a Poisson equation correction are much better. There is no seam either artifact, and the visual perception of the completed image is good.

\subsection{A PDE Assistant}

The novel model processes image based on exemplar. The block effects induced by the exemplar could be removed by using the Poisson equation. But when the target region is only a lathy linear structure, the block effects could not be attenuated by using the Poisson equation. Since the known information in the exemplar $\Psi_{H}$ is limited, it is not enough to correct the completion result. In this situation, we use a bi-directional diffusion PDE to assist the completion procedure. The PDE is used to complete the lathy linear structure, firstly. PDE will over-smooth the image and it cannot preserve textured information, so the novel exemplar-based model is then used in the preprocessed image to complete the large target region. The PDE model 
used in this paper is shown as follow and we will give some analyses about it.

$$
\begin{aligned}
& I(\xi)_{0}=I(\xi), \quad \omega_{0}=\Delta I(\xi)_{0}, \quad \mathbf{u}_{0}=\nabla^{\perp} I(\xi)_{0}, \\
& I(\xi)_{n+1}=I(\xi)_{n}+\Delta t \cdot(\nabla \cdot(\nabla I(\xi) /|\nabla I(\xi)|)), \\
& \omega_{n+1}=\Delta I(\xi)_{n+1}, \quad \mathbf{u}_{n+1}=\nabla^{\perp} I(\xi)_{n+1}, \\
& \omega_{n+2}=\omega_{n+1}-\Delta t \cdot\left(\mathbf{u}_{n+1} \cdot \nabla w_{n+1}\right), \\
& \Delta I(\xi)_{n+2}=\omega_{n+2} .
\end{aligned}
$$

There are diffusions along two orthogonal directions in Eq. (17): the gradient direction $\vec{n}$, where $\vec{n}=\nabla I(\xi)$; and the isophote direction $\vec{t}$, where $\vec{t}=\nabla^{\perp} I(\xi)$. The orthogonal directions are shown in Fig. 3 in which $x$ and $y$ denote the image coordinate system.

The equation used to assist the completion procedure is a bi-directional diffusion PDE, which reconstructs the image along two directions. The diffusion item along $\vec{n}$ is $(\nabla \cdot(\nabla I(\xi) /|\nabla I(\xi)|))$, and the diffusion item along $\vec{t}$ is $\left(\mathbf{u}_{n+1} \cdot \nabla w_{n+1}\right)$. So the PDE used in this paper has the non-linear topological sensitivity, and it reconstructs the target region differently along different geometric directions. The completed result of it is a smooth image and preserves the geometric structure well.

$(\nabla \cdot(\nabla I(\xi) /|\nabla I(\xi)|))$ equals to the curvature in image which is morphological invariant, so the stable status of TV model is adopted as the along gradient direction diffusion item directly. The detailed description of TV model is analyzed in Chan and Shen (2001). The along $\vec{t}$ diffusion item is derived according to the equations in fluid dynamics. The Navier-stokes $(\mathrm{N}-\mathrm{S})$ equation for uncompressible fluid is

$$
\rho \cdot(\mathrm{d} \mathbf{u} / \mathrm{d} t)=\rho \mathbf{f}-\nabla p+v \Delta \mathbf{u}, \quad \mathbf{u}=\nabla^{\perp} \Psi
$$

where $\mathbf{u}$ is fluid velocity, $\mathbf{f}$ is external force in fluid, $\rho$ is fluid density, $p$ is pressure, $v$ is viscosity coefficient, $\Psi$ is stream function in fluid. The curl result of both sides of N-S equation is

$$
\frac{\partial(\nabla \times \mathbf{u})}{\partial t}-\nabla \times(\mathbf{u} \times \omega)=\nabla \times f-\nabla \times\left(\frac{1}{\rho} \nabla p\right)+v \Delta(\nabla \times \mathbf{u}) .
$$

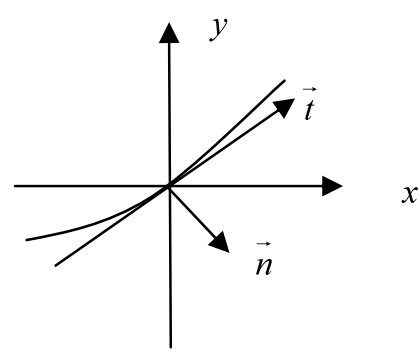

Fig. 3. Orthogonal diffused directions. 
The vorticity continuity equation is

$$
\nabla \cdot \omega=\nabla \cdot(\nabla \times \mathbf{u})=0 .
$$

Expanding the curl part, we get the Helmholtz vorticity equation:

$$
\mathrm{d} \omega / \mathrm{d} t=\partial \omega / \partial t+(\mathbf{u} \cdot \nabla) \omega=(\omega \cdot \nabla) \mathbf{u}+\nabla \times f-\nabla \times\left(\frac{1}{\rho} \nabla p\right)+v \Delta \omega .
$$

In 2-D image domain, $(\omega \cdot \nabla) \mathbf{u}, \nabla \times f, \nabla \times(\nabla p / \rho)$ are zeros, so the Helmholtz vorticity equation can be simplified as

$$
\mathrm{d} \omega / \mathrm{d} t=v \Delta \omega .
$$

Used in image completion, the Helmholtz equation should maintain the inviscid property (Bertalmio et al., 2001). The inviscid Helmholtz vorticity equation is

$$
\mathrm{d} \omega / \mathrm{d} t=0
$$

$\mathrm{d} \omega / \mathrm{d} t$ is the material derivative of $\omega$. It is composed as

$$
\mathrm{d} \omega / \mathrm{d} t=\partial \omega / \partial t+(\mathbf{u} \cdot \nabla) \omega .
$$

Then the inviscid Helmholtz vorticity equation in 2-D image domain is

$$
\partial \omega / \partial t+(\mathbf{u} \cdot \nabla) \omega=0 \quad \Rightarrow \quad \partial \omega / \partial t=-(\mathbf{u} \cdot \nabla) \omega .
$$

The inviscid Helmholtz equation transports a smooth measure $\omega$ in fluid domain along the isophotes direction $\mathbf{u}$ and it is morphological invariant. So it is adopted as the along isophotes diffusion item in the completion model.

\section{Experiments}

To demonstrate the validity of our model, the completed result of it is compared with that of the model in Criminisi et al. (2004). The most important benefit of the exemplar-based model is that it restores the structure and texture information simultaneously. So, the testing images used in this paper have complex texture patterns and have some geometric structures to be reconnected.

Firstly, we give some experimental results to shown the validity of the operation which uses PDE to assist the completion procedure. There are two parts in Fig. 4 needing completion, and one part is a lathy linear structure. Fig. 4(c) is the completed result of the novel exemplar-based model. All the textured information about cloud, trees, and seashore are restored. But the lathy default parts in the trees are not correctly preserved. 


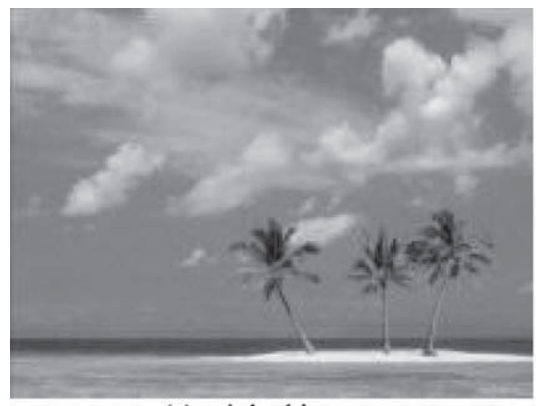

(a) original image

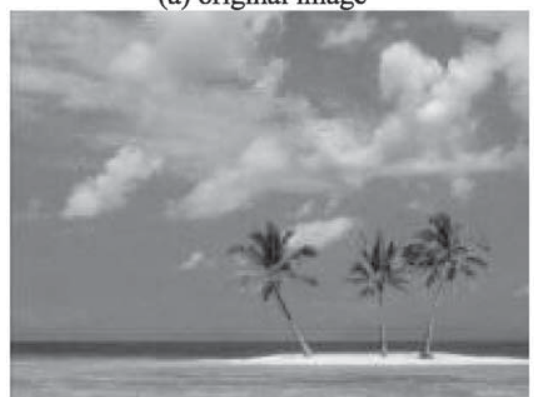

(c) result of the exemplar-based model

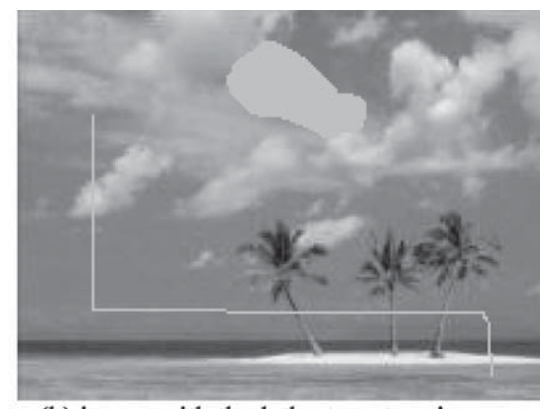

(b) image with the lathy target region

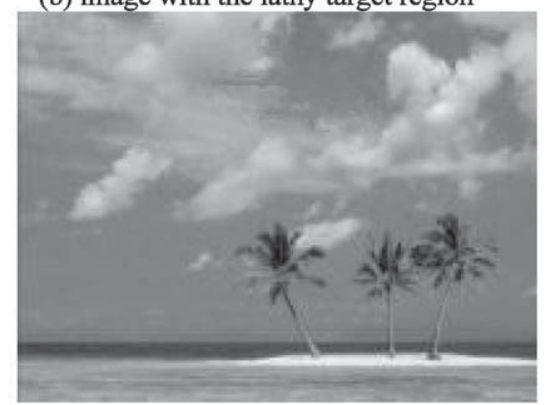

(d) result of the model with a PDE assistant

Fig. 4. Image completion in a natural image with different target regions.

Fig. 4(d) is the completed result of the model which uses a PDE assistant. It is clear the lathy parts of the target region are preserved well. There is textured information about cloud in the large part of target region in Fig. 4(b). After preprocessed by the PDE, this part in Fig. 4(d) is still completed by the novel exemplar-based model. So the lathy part of Fig. 4(d) is completed by the assistant of a PDE, and the visual perception of it is better than that of Fig. 4(c).

Another experiment is given in Fig. 5. We only select lathy linear structure parts in these images to complete. Fig. 5(c) is completed result of the novel exemplar-based model. Since the known information in the lathy part is limited, there are severe block effects in these images. Fig. 5(d) is the result of the model which uses a PDE assistant. Geometric information in the lathy part is well preserved and no block effect is generated.

Fig. 6(a) is a photo of Elephant Trunk Hill in Guilin, China. There are some boats and people in this image and they degrade the whole scene. They are marked out in Fig. 6(b). In Fig. 6(c), which is the completed result of the model in Criminisi et al. (2004), the surface of the lake is error filled. The structure of the lakeshore is not preserved and the inverted reflection of the Elephant Trunk Hill in water is filled with some parts of the lakeshore. The visual perception of Fig. 6(d), which is the completed result of our model, is good.

The geometric structure of lakeshore and the textured information about the surface of the lake are both restored well. Since a Poisson equation is used, no error texture pattern is filled in the target region and there is no artifact. There is no block effect in image either, and the reflection in the water is restored authentically. 


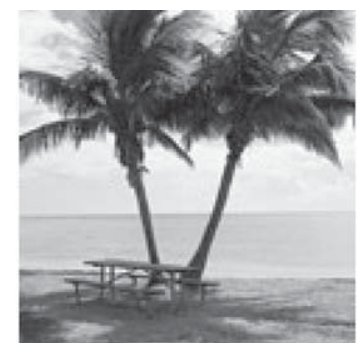

(a) original image

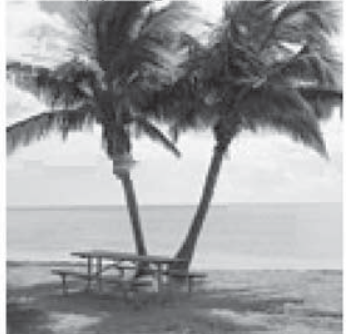

(c) result the of exemplar-based model

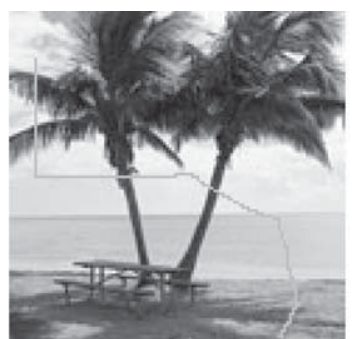

(b) image with the lathy target region

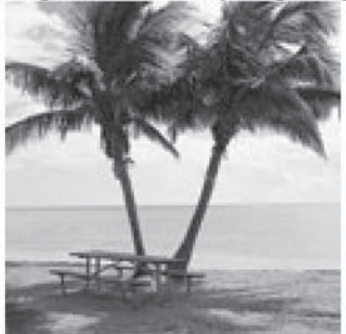

(d) result with a PDE assistant

Fig. 5. Image completion in natural image with lathy target region.

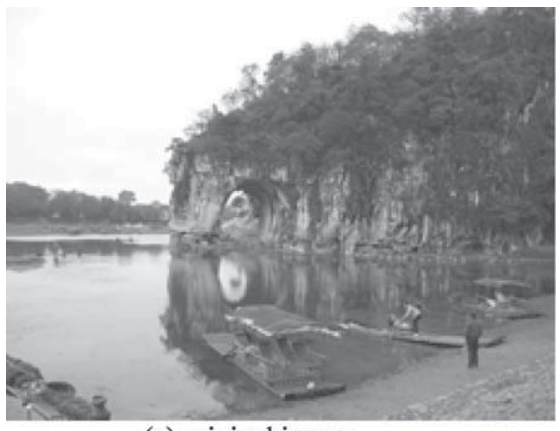

(a) original image

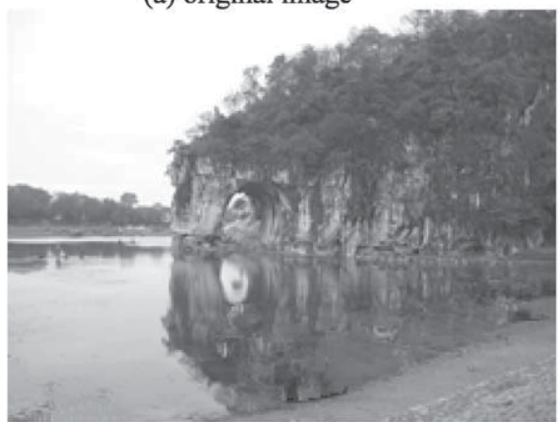

(c) completed result of model in Criminisi et al. (2004) (d) the completed result of our model

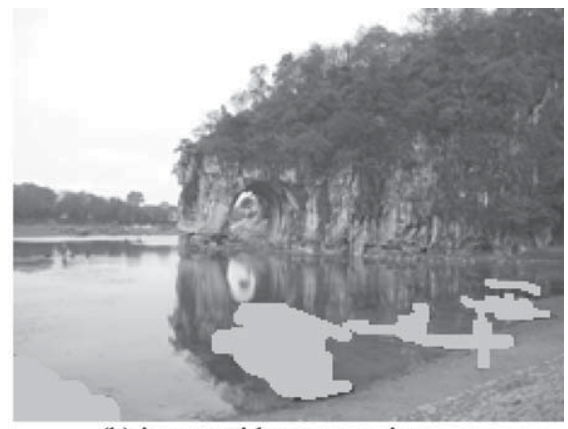

(b) image with target region

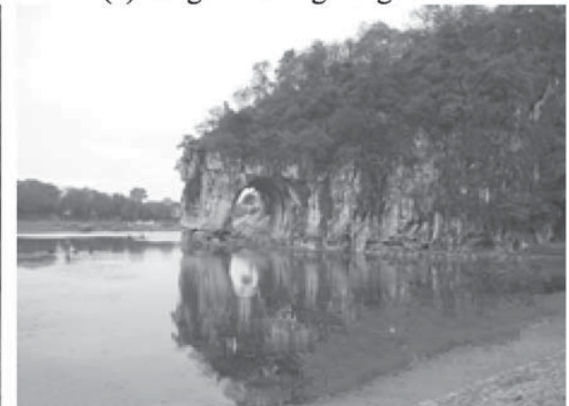

Fig. 6. The photo of Elephant Trunk Hill processed by different exemplar-based completion models. 
Table 1

SNR data of different images

\begin{tabular}{llll}
\hline & Fig. 4 & Fig. 5 & Fig. 6 \\
\hline Exemplar-based model & 18.5656 & 16.5559 & 22.5438 \\
The model using a PDE assistance & 19.1978 & 22.6270 & 32.1332
\end{tabular}

The SNRs of the completed images are given in Table 1. The SNRs of the images which are the results of the model using a PDE assistant are larger than that without PDE assistant. This is because the block effects caused by the exemplar-based model badly impact the visual perception of the image. The subjective results in Figs. 4-6, and the objective results in Table 1 demonstrate that when the target region is a lathy linear structure, using a PDE assist the completion procedure could improve the completion result of the novel exemplar-based model proposed in this paper.

The geometric structure preserving property of our model is further demonstrated in Fig. 7. Fig. 7(c) is the completed result of the model in Criminisi et al. (2004). The riverbank in Fig. 7(c) is propagated into the leg part of the target region, and the geometric structure of roof filled with leaves is breached. The completed result is not authentic. Fig. 7(d) is the result of our model. In this image, the riverbank is completed with a more plausible riverside and the linear structure of the roof is preserved well. It is accepted the image processed by our model is better, because the roof is never built with parts of leaves and the riverbank hardly has a suddenly extrusive part as that in Fig. 7(c).

Different images above show that the visual perception of the image processed by our model is best. In order to give an objective evaluation, we redo some experiments on these images. This time, we select the target regions in original un-damaged image, that is, the information in these regions are known, while we try to reproduce it by using the completion models. So it is possible to compare the completed images with original ones and compute the SNRs of different processed images. Table 2 gives SNRs of images which

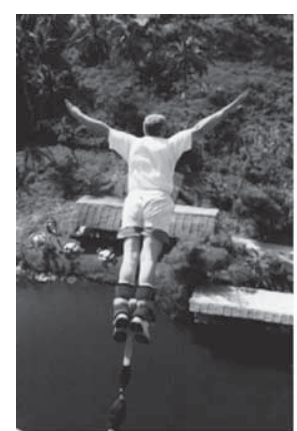

(a)

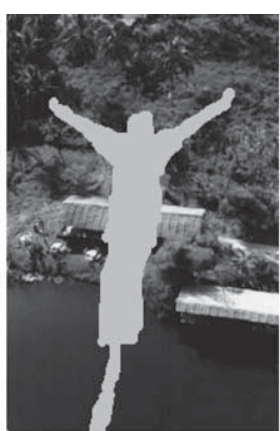

(b)

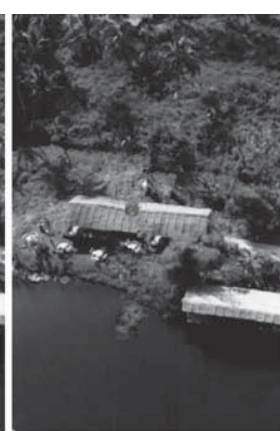

(c)

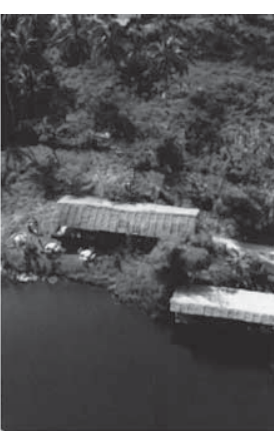

(d)

Fig. 7. The jumping images processed by different exemplar-based completion models. (a) original image; (b) image with the target region; (c) the completed result of model in Criminisi et al. (2004); (d) the completed result of our model. 
Table 2

SNRs of different images processed by different exemplar-based image completion models

\begin{tabular}{|c|c|c|}
\hline \multirow{2}{*}{$\begin{array}{l}\text { Original } \\
\text { image }\end{array}$} & \multicolumn{2}{|c|}{ Different processing models } \\
\hline & $\begin{array}{l}\text { The model in reference } \\
\text { (Criminisi } \text { et al., 2004) }\end{array}$ & $\begin{array}{l}\text { The novel model proposed } \\
\text { in this paper }\end{array}$ \\
\hline \multirow[t]{2}{*}{ Fig. 5} & 19.6260 & 21.5386 \\
\hline & 25.9475 & 28.5763 \\
\hline \multirow[t]{2}{*}{ Fig. 6} & 22.0350 & 22.4175 \\
\hline & 18.1368 & 20.5876 \\
\hline \multirow[t]{2}{*}{ Fig. 7} & 15.6141 & 16.4067 \\
\hline & 10.5872 & 11.6740 \\
\hline \multirow[t]{2}{*}{ Image Lena } & 12.4825 & 12.8246 \\
\hline & 15.7469 & 17.2837 \\
\hline \multirow[t]{2}{*}{ Image Barbala } & 24.6451 & 25.7702 \\
\hline & 20.1876 & 23.5891 \\
\hline
\end{tabular}

are processed by different completion models. We add two famous testing images "Lena" and "Barbala" into the experiments. The SNRs of the images which are processed by our model are larger than the SNRs of the images processed by the model in Criminisi et al. (2004). Our model has a better structure information preserving property, and the results of our model have no seams, block effects and jaggies. Both the subjective and objective experimental results in this section demonstrate the validity of the novel exemplar-based model proposed in this paper.

\section{Conclusions}

In this paper a novel exemplar-based image completion model is proposed. The novel model uses a composite function to determine the processing order of exemplar. The composite function is chosen and adopted according to both the color property and structure property of image. A cross-isophote diffused PDE is adopted in the composite function; therefore, an exemplar along a geometric structure has a higher processing priority and would be resorted prior to other exemplars to preserve the linear structure. The model has a good geometric structure preserving property. The size of exemplar is adaptively determined by the textured information, so the model could properly restore more texture patterns. The Poisson equation which is adopted in our model reduces seams, block effects, and artifacts left in the completed image, so the visual perception of the image which is processed by our model is much better than that of the image processed by the conventional model.

When the target region is a lathy linear structure, the exemplar-based model would induce severe block effects since the known information for the Poisson equation is limited. In this paper, a bi-directional diffusion PDE is used to assist the completion procedure. PDE has a better geometric structure preserving property and there is no block effect in 
the completed image. The novel model uses PDE to restore the complex geometric structures and uses the exemplar-based model to restore the complex textured information. It could be well used in many kinds of natural image completion.

\section{Acknowledgement}

The authors are grateful to the anonymous referees for their constructive and helpful comments. This paper is partly supported by the National Natural Science Foundation of China (No. 60472033, No. 60672062), the National Grand Fundamental Research 973 Program of China (No. 2004CB318005), the Technological Innovation Fund of Excellent Doctorial Candidate of Beijing Jiaotong University (No. 48026).

\section{References}

Bertalmio, M. (2006). Strong-continuation, contrast-invariant inpainting with a third-order optimal PDE. IEEE Transactions on Image Processing, 15(7), 1934-1938.

Bertalmio, M., Bertozi, A.L., Sapiro, G. (2001). Navier-Stokes, fluid dynamics and image and video inpainting. In: Proceedings of International Conference on Computer Vision and Pattern Recognition, Hawaii, USA, pp. 355-362.

Bertalmio, M., Sapiro, G., Caselles, V., Ballester, C. (2000). Image inpainting. In: Proceedings of ACM Conference in Computer Graphics (SIGGRAPH), New Orleans, LA, pp. 417-424.

Bornard, R., Lecam, E., Laborelli, L. (2002). Missing data correction in still images and image sequences. In: Proceedings of ACM. Multimedia, Juan-les-Pins, France, pp. 355-361.

Chan, T.F., Shen, J. (2001). Mathematical models for local nontexture inpaintings. SIAM Journal on Applied Math, 62(3), 1019-1043.

Criminisi, A., Perez, P., Toyama, K. (2004). Region filling and object removal by exemplar-based image inpainting. IEEE Transactions on Image Processing, 13(9), 1200-1212.

Drori, cohen-Or D., Yeshurun, H. (2003). Fragment-based image completion. ACM Transactions on Graphics, 22(3), 303-312.

Efros, A.A., Leung, T. (1999). Texture synthesis by nonparametric sampling. In: Proceedings of International Conference in Computer Vision, Greece, 1999, pp. 1033-1038.

Efros, A.A., Freeman, W.F. (2001). Image quilting for texture synthesis and transfer. In: Proceedings of ACM Conference in Computer Graphics (SIGGRAPH), Los Angeles, pp. 341-346.

Harrison, P. (2001). A non-hierarchical procedure for re-synthesis of complex textures. In: Proceedings of International Conference in Central Europe Computer Graphics and Visualization, Plzen, Czech Republic, pp. 190-197.

Komodakis, N., Tziritas, G. (2006). Image completion using global optimization. In: Proceedings of Computer Vision and Pattern Recognition, New York, pp. 442-452.

Ng, T.Y., Wen, C., Tan, T.S., Zhang, X., Kim, Y.J. (2005). Generating an $w$-tile set for texture synthesis. In: Proceedings of the 23rd Computer Graphics International, pp. 177-184.

Perez, P., Gangnet., M., Blake, A. (2004). PatchWorks: example-based region tiling for image editing. Technical Report, Microsoft Research, MSR-TR-2004-04.

Sun, J., Yuan, L., Jia, J., Shum, H.Y. (2005). Image completion with structure propagation. ACM Transactions on Graphic, 24(3), 861-868.

Tran, M., Datta, A. (2003). Synthesizing textures using variable neighborhood searching. In: Proceedings of the 7th International Conference on Digital Image Computing - Techniques and Applications, Sydney, pp. 643-652.

Wu, J.Y., Ruan, Q.Q., An, G.Y. (2009). A joint-diffused inpainting model for underexposure image preserving the linear geometric structure. Informatica, 20(1), 151-163. 
J. Wu was born in Hebei, China in 1982. She received the BS degree in computer science and technology from Beijing Jiaotong University, Beijing China in Jun. 2004, and the Ph.D. degree in signal processing from Beijing Jiaotong University in Jan. 2009. She is now a Postdoctoral Fellow at the Department of Electrical and Computer Engineering of the Hong Kong University of Science and Technology (HKUST). Her research interests include image processing, partial differential equation, image recognition, etc.

Q. Ruan was born in 1944. He received the BS and MS degrees from Northern Jiaotong University, China in 1969 and 1981 respectively. From January 1987 to May 1990, he was a visiting scholar in the University of Pittsburgh, and the University of Cincinnati. Subsequently, he has been a visiting professor in USA for several times. He has published 2 books and more than 100 papers, and achieved a national patent. Now he is a professor, doctorate supervisor of the Institute of Information Science, Beijing Jiaotong University. His main research interests include digital signal processing, computer vision, pattern recognition, and virtual reality etc. 


\title{
Pavyzdžiu grịstas vaizdu atstatymas naudojant diferencialines lygtis su dalinėmis išvestinėmis
}

\author{
Jiying WU, Qiuqi RUAN
}

Siūlomas modelis tinkamai atstato vaizdą be užlankų, blokinio tašku intensyvumų efekto ar kitu iškraipymų. Pirmiausia, siūlomas modelis nustato pavyzdžio apdorojimo tvarką atsižvelgiant i kompozicinę funkciją, kuri įvertina vaizdo taškų spalvines bei struktūros savybes. Tada atsižvelgiant i geometrinę struktūrą apdorojamas pavyzdys. Antrajame žingsnyje CIELAB spalvinèje erdveje randamas panašiausias pavyzdžio atitikmuo, kurio dydis adaptyviai nustatomas iš lokalios tekstūros informacijos. Trečiajame žingsnyje Poisono lygtis taikoma užlankų, blokinio tašku intensyvumo efektui ar kitiems iškraipymams šalinti, kuris atsiranda po pavyzdžiu grịsto atstatymo. Galiausiai tiesinès struktūros apdorojamos panaudojus dvikryptę difuziją apskaičiuotą iš diferencialiniu lygčiu su dalinèmis išvestinèmis. Eksperimentiniai rezultatai rodo, kad naujasis modelis gali tinkamai atstatyti pažeistą vaizdo sritị išlaikant jo geometrinę struktūrą be blokinio tašku intensyvumo efekto, kas apsprendžia jo privalumą prieš kitus pavyzdžiu grịstus vaizdų atstatymo modelius. 